\title{
ORIGINAL
}

\section{COMPARACIÓN DE LOS BAREMOS ESPAÑOL, FRANCES Y ALEMÁN PARA MEDIR LA DEPENDENCIA DE LAS PERSONAS CON DISCAPACIDAD Y SUS PRESTACIONES}

\author{
Irene Albarrán Lozano (1), Pablo Alonso González (2) y Catalina Bolancé Losillas (3) \\ (1) Departamento de Estadística. Universidad Carlos III de Madrid. \\ (2) Departamento de Estadística, Estructura Económica y OEI Universidad de Alcalá. \\ (3) Departamento de Econometría, Estadística y Economía Española. Universidad de Barcelona.
}

\section{RESUMEN}

Fundamento: A pesar del consenso generalizado sobre lo que es una persona dependiente, su puesta en práctica en cada país arroja resultados completamente diferentes. El objetivo de este trabajo es comparar la aplicación de distintos baremos de valoración de la dependencia sobre la población la española y apreciar las diferencias existentes en sus resultados sobre el derecho a recibir ayudas.

Método: Se aplican las escalas de valoración de la dependencia de Francia, Alemania y España sobre la población española reflejada en la EDDES de 1999 comparando el sistema español con cada uno de los otros dos usando el análisis factorial de correspondencias.

Resultados: Según LA EDDES, el número de personas dependientes asciende, según a 1.398.767. Aplicando el baremo español 776.475 recibirían algún tipo de ayuda pública, con el baremo alemán serían 745.520 y con el baremo francés 315.514. Los resultados del análisis de correspondencias muestran cómo el baremo español posee mayor semejanza con el alemán que con el francés (los coeficientes de correlación de Spearman y de Kendall se reducen casi en un $40 \%$ cuando comparamos el sistema español con el francés).

Conclusiones: A igualdad de características personales, la utilización de uno u otro modelo condiciona tanto la catalogación como persona dependiente como la posibilidad de recibir ayudas públicas. Por número de beneficiarios, el más generoso es el sistema español y el más restrictivo el francés, siendo este último el único en el que la edad es una variable limitativa.

Palabras clave: Persona con discapacidad. Análisis factorial. España. Francia. Alemania.

Correspondencia:

Irene Albarrán Lozano

Departamento de Estadística. Universidad Carlos III de Madrid.

Facultad de Ciencias Sociales y Jurídicas

Avda Universidad Carlos III 22

Colmenarejo 28270, Madrid

correo electrónico: ialbarra@est-econ.uc3m.es

\section{ABSTRACT}

\section{A comparison of the Spanish, the French and the German valuation Scales to Measure Dependency and Public Support for People with Disabilities}

Background: Even if there is a wide consensus on the concept of a dependent individual, in practice national valuation systems produce completely different results. The objective in this work is to compare the application of different valuation scales for dependency on the Spanish population and to identify the differences in the results on the right to obtain public support.

Methods: The evaluation definition and schemes that are applied in France, Germany and Spain are implemented to the Spanish population using data from the Survey on Disabilities, Dependency and Health Status. The Spanish system is compared to the other two using factorial correspondence analysis.

Results: According to the survey the total number of dependent individuals in the population is 1.398.767. Under the Spanish scale, 776.475 would receive some type of public support, under the German scale 745.520 would qualify and when applying the French scale, those would be 315.514 . Correspondence analysis results show that the Spanish scale is much more similar to the German scale than it is to the French scale. (Spearman's correlation coefficient and Kendall's are reduced by almost $40 \%$ when the Spanish scale is compared to the French scale, rather than the German scale).

Conclusions: With the same personal conditions, the system used influences both the degree of severity of dependence and the possibility to become eligible to public funds. The Spanish system is the most generous and the French system is the most restrictive one, the latter also imposes limitations on age.

Key words: Disabled persons. Factor Analysis, Statistical. Spain. France. Germany.

Los autores agradecen las ayudas del Ministerio de Educación y Ciencia y el Plan Nacional de Investigación Científica, Desarrollo e Innovación Tecnológica a través del proyecto SEJ2005-08070/ECON cofinanciado con fondos FEDER, del Ministerio de Ciencia e Innovación y los fondos FEDER con el proyecto ECO2008-01223/ECON y del Instituto de Mayores y Servicios Sociales (IMSERSO, Ministerio de Trabajo y Asuntos Sociales) a través del proyecto 99/07. 


\section{INTRODUCCIÓN}

Durante las últimas décadas las sociedades europeas han experimentado un progresivo y acusado proceso de envejecimiento. Según la Comisión Europea ${ }^{1}$ entre las razones de esta situación destacan: el aumento de la longevidad debido a los avances médicos y a las mejoras en la calidad de vida, las bajas tasas de natalidad a causa de las dificultades para encontrar trabajo, el coste de la vivienda, el retraso para tener hijos y la incorporación de la mujer al mercado laboral, entre otras.

Alemania, Francia y España son tres de los países que más han acusado este proceso de envejecimiento. Según datos de la OMS, en el caso alemán las personas de la tercera edad suponían el 17,5\% de su población en 2003, esperándose que representen el $26,4 \%$ en $2030^{2}$. Por su parte, en 2004 el $16 \%$ de la población francesa tenía más de 65 años, esperándose que el peso de este grupo de población llegue al $24 \%$ en $2030^{3}$. En cuanto a nuestro país, el peso de este segmento de población sobre el total de 2004 era del 16,8\%, esperándose que llegue hasta el $30,8 \%$ en $2050^{4}$. En concreto, según Naciones Unidas, en 2050 España será el país más envejecido del mundo 5 .

En este trabajo se analizan y comparan los sistemas de dependencia de Alemania, Francia y España. Aunque no entramos en la valoración de los costes de la dependencia en los distintos países, uno de los aspectos que justifica la importancia de este análisis es que para comparar los costes de los diferentes sistemas de dependencia un primer paso necesario es aplicarlos sobre una misma población y analizar que individuos se ven más o menos beneficiados por los distintos sistemas.

El envejecimiento demográfico no sólo afecta a los países europeos sino que se trata de una situación generalizada, sobre todo en los países desarrollados. Además, se prevé un aumento de la población mayor (especialmente de las personas mayores de 80 años de edad) ${ }^{6}$ que hará necesario destinar mayores recursos para la atención y los cuidados sanitarios de las generaciones más longevas, una redistribución de recursos intergeneracional, un aumento de los llamados cuidados formales e informales $y$, en definitiva, una mayor importancia de la llamada vejez débil (frail elderly), es decir, las personas que debido a enfermedades o discapacidades asociadas a la edad necesitan, varias veces al día, ayuda para realizar las actividades cotidianas que incluyen: levantarse, asearse, vestirse, ir al servicio, comer, cocinar, salir de casa y tener vida social.

Los cambios demográficos y sociales están provocando una expansión de las necesidades de cuidados de larga duración debido, entre otros aspectos, a la reducción del tamaño de las familias y la incorporación de la mujer a la actividad laboral, que han propiciado una reducción de los cuidados informales y el consiguiente aumento de los cuidados formales que deben ser gestionados ${ }^{7,8}$.

Se establece claramente una estrecha y directa relación entre envejecimiento, aumento de los gastos sanitarios y el papel a desarrollar tanto por los seguros públicos como los privados en la cobertura de los servicios demandados ${ }^{9}$. Además, existen varios estudios ${ }^{10-13}$ que sugieren un aumento de los gastos sanitarios en las próximas décadas, siendo una de las razones principales el proceso de envejecimiento de la población que están experimentando los países desarrollados. Así, uno de los fenómenos asociados es el aumento del número de personas con dependencia a causa de la vejez. En consecuencia, la dependencia asociada al envejecimiento se convierte en un problema socio-sanitario y económico ${ }^{14}$.

Se entiende por persona dependiente aquélla que requiere de la ayuda prolongada de otra persona para llevar a cabo los 
actos esenciales de la vida cotidiana. Una definición más precisa se recoge en la Recomendación del Consejo de Europa relativa a la dependencia ${ }^{15}$.

En Alemania se considera que una persona requiere cuidados ligados a una situación de dependencia si padece enfermedad física, psíquica o mental, o sufre discapacidad por la que requiere apoyo significativo para realizar las tareas corrientes de la vida diaria durante, al menos, 6 meses ${ }^{16}$. En Francia, una persona dependiente es una persona de al menos 60 años, en situación de incapacidad debida a la falta o pérdida de autonomía ligada a una situación específica física o mental ${ }^{17}$. Finalmente, en España se define a las personas dependientes como aquéllas que, por diversas razones, precisan de la atención de terceras personas o ayudas importantes para realizar las tareas básicas de la vida diaria o, en el caso de que la persona padezca discapacidad intelectual o enfermedad mental, requiera de otros apoyos para su autonomía personal ${ }^{18}$.

Por tanto, la definición de persona dependiente aplicada en los tres países mantiene las características esenciales recogidas por el Consejo de Europa aunque Francia incorpore como novedad la delimitación relativa a la edad (mínimo 60 años). Sin embargo, aunque exista una definición de dependencia comúnmente aceptada por distintos países, cada uno utiliza criterios distintos para decidir qué individuos dependientes deben recibir ayuda por parte de las Administraciones Públicas, es decir, cada país diseña un baremo que permite determinar qué individuos dependientes deben recibir ayuda y cuál debe ser la cuantía de esta ayuda. Los baremos de distintos países muestran importantes diferencias, y su aplicación va a tener distintas consecuencias económicas. Además, un mismo individuo dependiente, según en el país en el que resida, tendrá derecho a ayuda económica o no y/o se le podrán otorgar o no determinadas prestaciones.
Existen trabajos que analizan si dentro de la población dependiente puede hablarse de homogeneidad ${ }^{19-21}$. Sin embargo, esta investigación se plantea dar respuesta a las preguntas sobre qué se entiende por persona en situación de dependencia con derecho a recibir ayudas y si son homogéneos los diversos criterios requeridos. En esta misma línea se analizarán, igualmente, las consecuencias derivadas de comparar las legislaciones de los tres países europeos mencionados (Alemania, Francia y España) al aplicarlos sobre una misma población: la española.

Este trabajo no compara globalmente los sistemas de cuidados enfocados a la atención de dependientes o personas mayores débiles, tal y como se hace en determinados estudios $^{22-27}$, sino que se centra en el análisis del impacto que tiene la aplicación de diferentes escalas nacionales de valoración sobre una misma población, para así estimar la sensibilidad del número de personas susceptibles de recibir ayudas derivadas de su condición de dependientes. Aún cuando parece existir un consenso sobre qué se considera persona en situación de dependencia, la puesta en práctica de los sistemas de atención a estas personas diverge de unos países a otros. La consecuencia inmediata es que una persona con determinadas discapacidades puede ser considerada beneficiaria de ayudas en un cierto país pero no serlo en otro. De hecho, la falta de coincidencia en los criterios de valoración no sólo ocurre al evaluar la dependencia, sino también, al analizar diversos indicadores de la discapacidad $^{28}$. Si nos centramos en estudios del fenómeno de ayuda a la dependencia, este resultado ha sido confirmado, además, al estudiar la valoración del grado de severidad de la dependencia y su influencia en la estimación de los costes de los servicios de cuidados de larga duración ${ }^{29}$.

La mayoría de los trabajos $13,22,23,25-27,29$ se centran en el estudio exclusivo de las personas mayores dependientes. En ellos se 
comparan los sistemas de cuidados existentes en varios países ${ }^{22-27}$, pero analizando los tipos de cuidados (médicos, personales y del hogar), servicios así como el apoyo financiero ofrecido, utilizando comparaciones a nivel global o estudiando casos particulares. Concluyen, entre otras cuestiones, que existen divergencias importantes entre los sistemas, aunque aparentemente el abanico de servicios propuestos sea muy semejante.

El objetivo de este trabajo es comparar los diferentes baremos de valoración de la dependencia en Francia, Alemania y España con el fin de señalar las diferencias en la definición de persona dependiente y en las prestaciones que cada uno de ellos reconoce.

\section{MATERIAL Y MÉTODO}

En el Libro Blanco de la Dependencia ${ }^{30}$ se describen en detalle los sistemas alemán y francés. El sistema español se aborda en la Ley 39/ 2006.

En Alemania el sistema de dependencia se materializa en un Seguro de Dependencia, mediante la entrada en vigor de la Ley del Seguro de Cuidados en enero de 1995. En este país, para determinar el grado de dependencia ${ }^{16}$ se consideran cuatro ámbitos que condicionan la vida diaria: 1) aseo personal, 2) alimentación (preparar y tomar la comida), 3) movilidad y 4) realizar las tareas domésticas. Como representamos en la tabla 1 los niveles de dependencia (N1, N2 y N3) se fijan en función de la frecuencia de veces que el individuo necesita ayuda en los cuatro ámbitos citados con anterioridad.

El actual sistema francés se establece mediante la ley 2001-647, que desarrolla lo que vendrá a llamarse Prestación Personalizada de Autonomía, que incorpora el requisito de la edad (ser mayor de 60 años) para tener derecho a ayuda. En Francia para clasificar a los individuos en niveles de dependencia se utiliza la escala AGGIR (Gerontological Autonomy IsoResources Group), que se basa 17 variables, 10 de ellas son determinantes para establecer si procede o no dar las ayudas y las otras 7 son ilustrativas del grado de dependencia doméstica y social. Cada una de estas variables puede tomar los valores $\mathrm{A}, \mathrm{B}$ o $\mathrm{C}$, dependiendo de si puede realizar la tarea de forma correcta, irregular o no la puede realizar. A partir de las variables citadas con anterioridad se determina la escala denominada GIR (Iso-Resources Gruops), que clasifica a la población dependiente en 6 grupos (del GIR 1 al GIR 6), cuyas características describimos en la tabla 2. Aunque, al igual que el sistema alemán, el sistema francés tiene en cuenta la frecuencia con la que el dependiente necesita ayuda para sus cuidados, este hecho no se cuantifica de forma tan evidente.

Finalmente, el sistema español se materializa con la Ley 39/2006, que establece que la situación de dependencia se clasificará en tres grados (Grado I, Grado II y Grado III) (tabla 2). En España el sistema

Tabla 1

Comparación de los sistemas de valoración de la dependencia en Alemania

\begin{tabular}{|c|c|c|c|c|c|}
\hline \multirow{2}{*}{\multicolumn{2}{|c|}{ Grupos }} & \multicolumn{4}{|c|}{ Necesidad de Ayuda } \\
\hline & & 1) Aseo personal & 2) Alimentación & 3) Movilidad & 4) Tareas domésticas \\
\hline \multirow{3}{*}{ 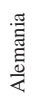 } & N1 & \multicolumn{3}{|c|}{ Una vez al día, al menos 90 minutos, 45 en cuidados básicos } & Varias veces por semana \\
\hline & N2 & \multicolumn{3}{|c|}{$\mathrm{Al}$ menos tres veces al día en distintas horas, con una duración mínima de 3 horas, 2 en cuidados básicos } & Varias veces por semana \\
\hline & $\mathrm{N} 3$ & \multicolumn{3}{|c|}{ Asistencia permanente durante al menos 5 horas diarias, 4 en cuidados básicos } & Varias veces por semana \\
\hline
\end{tabular}

Fuente: elaboración propia a partir de las leyes y los baremos nacionales ${ }^{16}$. 
Tabla 2

Comparación de los sistemas de valoración de la dependencia en Francia y España

\begin{tabular}{|l|l|l|}
\hline País & Grupo & Necesidad de Ayuda \\
\hline \multirow{5}{*}{ Francia } & GIR 1 & $\begin{array}{l}\text { Personas postradas en la cama o en sillas de ruedas, que han perdido su independencia mental, física o motriz y precisan de ayuda } \\
\text { continuada de sus cuidadores }\end{array}$ \\
\cline { 2 - 3 } & GIR 2 & $\begin{array}{l}\text { Personas postradas en la cama o en sillas de ruedas, cuya capacidad mental no está completamente afectada y necesitan asistencia para } \\
\text { la mayoría de sus actividades diarias (también se incluyen aquí a personas con las capacidades mentales afectadas pero no las físicas) }\end{array}$ \\
\cline { 2 - 3 } & GIR 4 & Individuos que poseen autonomía mental, que tienen autonomía motriz parcial, pero necesitan ayuda diariamente varias veces al día \\
\cline { 2 - 4 } & GIR 5 & $\begin{array}{l}\text { Personas que no pueden asegurar que se incorporen por sí solas pero que, una vez levantados de la cama o de la silla, pueden } \\
\text { moverse (necesitan ayuda para levantarse y vestirse) }\end{array}$ \\
\cline { 2 - 4 } & GIR 6 & Personas que realizan las actividades determinantes para la dependencia por sí mismas \\
\hline \multirow{5}{*}{ España } & Grado I & Dependencia moderada. La persona necesita ayuda para realizar varias actividades básicas de la vida diaria, al menos una vez al día \\
\cline { 2 - 3 } & Grado II & $\begin{array}{l}\text { Dependencia severa. El individuo necesita ayuda para realizar varias actividades básicas de la vida diaria dos o tres veces al día, } \\
\text { pero no requiere la presencia permanente de un cuidador }\end{array}$ \\
\cline { 2 - 3 } & Grado III & $\begin{array}{l}\text { Gran dependencia. La persona necesita ayuda para realizar varias actividades básicas de la vida diaria varias veces al día y, por su } \\
\text { pérdida total de autonomía mental o física, necesita la presencia indispensable y continua de otra persona }\end{array}$ \\
\hline
\end{tabular}

Fuente: elaboración propia a partir de las leyes y los baremos nacionales ${ }^{17,18,31}$

de dependencia establece los niveles a partir de la frecuencia en la necesidad de ayuda pero, al igual que el sistema francés, no los presenta de un modo tan cuantificado como en el sistema alemán. En cada uno de los grados de dependencia se establecerán dos niveles, en función de la autonomía de las personas y de la intensidad del cuidado que requieren. A efectos de su valoración los grados y niveles de dependencia se determinan mediante la aplicación del baremo aprobado en el Real Decreto 504/2007, de 20 de abril, debiéndose obtener al menos, 25 puntos para tener derecho a recibir ayudas públicas. A partir de esta cota por cada 25 puntos adicionales se asciende de grado. Dentro de cada uno de los grados los 15 primeros puntos ubican al individuo en el nivel $1 \mathrm{y}$ los siguientes en el nivel 2.

Para llevar a cabo la comparación de los tres sistemas descritos se utilizaron los datos de la Encuesta de Discapacidades, Deficiencias y Estados de Salud (EDDES), referidos a personas mayores de cinco años. Esta encuesta fue elaborada por el Instituto Nacional de Estadística (INE) en 1999 siguiendo la Clasificación Internacional de Deficiencias, Discapacidades y Minusvalías de 1980 (CIDDM-1980), la cual identifica 36 discapacidades agrupa- das en 10 bloques. Cada discapacidad lleva emparejado su grado de severidad, el cual puede ser moderado, severo o total. La tabla 3 recoge la CIDDM-1980, señalándose las discapacidades consideradas por cada sistema.

La aplicación de los sistemas de dependencia de cada uno de los tres países sobre los datos de la EDDES, para su posterior comparación, permite determinar cómo sería la composición de la muestra de personas dependientes en cada sistema. Para ello es necesario definir qué se entiende por persona dependiente y persona dependiente con derecho a ayudas en cada uno de los sistemas considerados. Una vez superada esta fase, se procedió a clasificar a la población mayor de cinco años recogida en la EDDES según cada uno de los tres sistemas para, finalmente, realizar las comparaciones dos a dos entre los resultados de aplicar el baremo español con los correspondientes a aplicar el alemán y el francés.

Por lo que respecta a la definición de persona dependiente es bastante similar en los tres países, por ello creemos que puede tomarse como válida en los tres casos la definición dada por el Consejo de Europa, 
Tabla 3

CIDDM-1980 y discapacidades consideradas en cada baremo nacional

\begin{tabular}{|c|c|c|c|c|}
\hline Tipo & Discapacidad & Alemania & Francia & España \\
\hline \multirow{4}{*}{ Ver } & 1: Discapacidad para recibir cualquier imagen & & & \\
\hline & 2: Discapacidad para tareas visuales de conjunto & & & \\
\hline & 3: Discapacidad para tareas visuales de detalle & & & \\
\hline & 4: Otras discapacidades de la visión & & & \\
\hline \multirow{3}{*}{ Oír } & 5: Discapacidad para recibir cualquier sonido & & & \\
\hline & 6: Discapacidad para la audición de sonidos fuertes & & & \\
\hline & 7: Discapacidad para escuchar el habla & & & \\
\hline \multirow{4}{*}{ Comunicarse } & 8: Comunicarse a través del habla & & $\mathrm{X}$ & \\
\hline & 9: Comunicarse a través de lenguajes alternativos & & $\mathrm{X}$ & \\
\hline & 10: Comunicarse a través de gestos no signados & & $\mathrm{X}$ & \\
\hline & 11: Comunicarse a través de escritura-lectura convencional & & $\mathrm{X}$ & \\
\hline \multirow{4}{*}{$\begin{array}{l}\text { Aprender, aplicar } \\
\text { conocimientos } \\
\text { y desarrollar tareas }\end{array}$} & 12: Reconocer personas, objetos y orientarse en el espacio & & $\mathrm{X}$ & $\mathrm{X}$ \\
\hline & 13: Reconocer informaciones y episodios recientes y/o pasados & & $\mathrm{X}$ & \\
\hline & 14: Entender y ejecutar órdenes sencillas y/o realizarlas & & & $\mathrm{X}$ \\
\hline & 15: Entender y ejecutar órdenes complejas y/o realizarlas & & & \\
\hline \multirow{3}{*}{ Desplazarse } & 16: Cambios y mantenimiento de las diversas posiciones del cuerpo & $\mathrm{X}$ & $\mathrm{X}$ & $\mathrm{X}$ \\
\hline & 17: Levantarse, acostarse, permanecer de pie o sentado & $\mathrm{X}$ & $\mathrm{X}$ & $\mathrm{X}$ \\
\hline & 18: Desplazarse dentro del hogar & $\mathrm{X}$ & $\mathrm{X}$ & $\mathrm{X}$ \\
\hline \multirow{3}{*}{ Utilizar brazos y manos } & 19: Trasladar-transportar objetos no muy pesados & & & \\
\hline & 20: Utilizar utensilios y herramientas & & & \\
\hline & 21: Manipular objetos pequeños con manos y dedos & & & \\
\hline \multirow{3}{*}{$\begin{array}{l}\text { Desplazarse fuera } \\
\text { del hogar }\end{array}$} & 22: Deambular sin medio de transporte & $\mathrm{X}$ & $\mathrm{X}$ & $\mathrm{X}$ \\
\hline & 23: Desplazarse en transportes públicos & & & $\mathrm{X}$ \\
\hline & 24: Conducir vehículo propio & & & $\mathrm{X}$ \\
\hline \multirow{4}{*}{ Autocuidado } & 25: Asearse solo: lavarse y cuidarse de su aspecto & $\mathrm{X}$ & $\mathrm{X}$ & $\mathrm{X}$ \\
\hline & 26: Control de las necesidades y utilizar solo el servicio & $\mathrm{X}$ & $\mathrm{X}$ & $\mathrm{X}$ \\
\hline & 27: Vestirse, desvestirse, arreglarse & $\mathrm{X}$ & $\mathrm{X}$ & $\mathrm{X}$ \\
\hline & 28: Comer y beber & $\mathrm{X}$ & $\mathrm{X}$ & $\mathrm{X}$ \\
\hline \multirow{5}{*}{ Realizar tareas del hogar } & 29: Cuidarse de las compras y el control de suministros y servicios & $\mathrm{X}$ & & $\mathrm{X}$ \\
\hline & 30: Cuidarse de las comidas & $\mathrm{X}$ & & $\mathrm{X}$ \\
\hline & 31: Cuidarse de la limpieza y el planchado de la ropa & $\mathrm{X}$ & & $\mathrm{X}$ \\
\hline & 32: Cuidarse de la limpieza y el mantenimiento de la casa & $\mathrm{X}$ & & $\mathrm{X}$ \\
\hline & 33: Cuidarse del bienestar de los demás miembros de la familia & & & $\mathrm{X}$ \\
\hline \multirow{3}{*}{$\begin{array}{l}\text { Relaciones con otras } \\
\text { personas }\end{array}$} & 34: Mantener relaciones de cariño con familiares próximos & & & \\
\hline & 35: Hacer amigos y mantener la amistad & & & \\
\hline & 36: Relacionarse con compañeros, jefes y subordinados & & & \\
\hline
\end{tabular}

Fuente: elaboración propia a partir de EDDES y de los baremos nacionales.

la cual exige el cumplimiento de tres requisitos: que exista una limitación que merme ciertas capacidades, que la persona padezca incapacidad para realizar las actividades de la vida diaria y que necesite de la ayuda de una tercera persona.

En España las personas dependientes con derecho a ayuda son las que se definen en la tabla 2, el contenido de esta tabla se termina de completar con el hecho de tener más de tres años y que, tras aplicar el baremo recogido en el RD 504/2007 31 , poseen una puntuación igual o mayor a 25. En Alemania, las personas dependientes con derecho a ayuda son las definidas en la tabla 1 agrupadas en tres niveles: N1, N2 y N3. Las personas dependientes con derecho a ayuda 
según el sistema francés son las que se sitúan en los niveles de GIR 1 a GIR 4 definidos en la tabla 2.

A partir de aquí se trata de estudiar si existía relación entre las clasificaciones obtenidas con los sistemas aplicados, para lo cual se estimaron los coeficientes de correlación de Spearman y el de Kendall. Finalmente, mediante el uso del paquete SAS, se realizaron dos análisis de correspondencias simples para representar conjuntamente las categorías de personas dependientes del sistema español con cada uno de los otros dos sistemas empleados. Esta técnica es similar ${ }^{32,33}$ al análisis de componentes principales, aunque adaptada al uso de variables categóricas. Permite representar en un mismo espacio ddimensional las categorías recogidas en filas y columnas de una tabla de contingencia, donde d es el mínimo valor entre el número de filas y el de columnas minorado en uno. Su uso hace posible encontrar las similitudes que puedan existir entre las diferentes categorías que se aplican en dos sistemas de clasificación. El estadístico ji-cuadrado, nos permite inferir si la correlación entre pares de sistemas es significativa.

\section{RESULTADOS}

La aplicación de los criterios definidos por el Consejo Europeo sobre la EDDES arrojó un número de 1.398 .767 personas dependientes en 1999, de las cuales 462.459 eran hombres y 936.308 mujeres. Sobre este conjunto de personas es sobre el que se realizaron las comparaciones. La tabla 4 ofrece el número (y el porcentaje que representa respecto al total de la población) de personas dependientes afectadas por cada una de las 36 discapacidades. Hay que tener en cuenta que una misma persona puede padecer más de una discapacidad por lo que la suma de los porcentajes supera el $100 \%$.
Tras aplicar el baremo español a las personas dependientes resultó que 622.292 tendrían una puntuación inferior a 25, por lo que no recibirían ayuda alguna; 384.077 estarían dentro del Grado I, 191.781 en el Grado II y 200.617 en el Grado III. Por tanto, según este baremo al 44,49\% de las personas dependientes no tendrían derecho a ayuda procedente de la administración.

Con el baremo alemán que 745.520 personas dependientes tenían derecho a algún tipo de ayuda y las $653.247(46,70 \%)$ restantes no la obtendrían.

Los resultados de la distribución de las personas dependientes entre los niveles del baremo alemán, junto con la comparación con el baremo español, se recogen en la tabla 5. El número total de personas que no teniendo derecho a ayuda según el baremo alemán (columna 0 en la tabla 5) alcanzaron un grado de dependencia I, II o III en el sistema español era de 163.000. La situación inversa también existe, es decir, si centramos nuestra atención en la fila 0 de la tabla 5, casi 132.000 personas no recibirían ayuda en España y sí en Alemania. También, habría 613.530 personas que, fuese cual fuese el sistema aplicado, mantendrían su condición de dependiente con derecho a ayudas.

Teniendo en cuenta que en Francia se impone una edad mínima de 60 años, de las 1.398.767 definidas como personas dependientes según los criterios del Consejo Europeo, serían dependientes 1.057.048 de las cuales 315.514 estarían en los niveles del GIR 1 al 4. Las personas dependientes que no recibirían ningún tipo de ayuda suponen el 77,44\%. Los resultados obtenidos, junto con la comparación del baremo español se recogen en la tabla 6. En este caso, si centramos nuestra atención en las columnas GIR 0, GIR 6 y GIR 5, las cuáles identifican a las personas que no percibirían ayuda según el sistema francés, se observa que de un total 1.083.000 personas depen- 
Tabla 4

Número y porcentaje de personas dependientes afectadas por cada discapacidad

\begin{tabular}{|c|c|c|c|}
\hline Tipo & Discapacidad & Número de personas & $\%$ \\
\hline \multirow{4}{*}{ Ver } & 1 & 44.273 & 3,2 \\
\hline & 2 & 184.745 & 13,2 \\
\hline & 3 & 226.595 & 16,2 \\
\hline & 4 & 93.760 & 6,7 \\
\hline \multirow{3}{*}{ Oír } & 5 & 27.660 & 2,0 \\
\hline & 6 & 65.206 & 4,7 \\
\hline & 7 & 226.580 & 16,2 \\
\hline \multirow{4}{*}{ Comunicarse } & 8 & 93.068 & 6,7 \\
\hline & 9 & 70.553 & 5,0 \\
\hline & 10 & 57.828 & 4,1 \\
\hline & 11 & 314.752 & 22,5 \\
\hline \multirow{4}{*}{ Aprender, aplicar conocimientos y desarrollar tareas } & 12 & 179.066 & 12,8 \\
\hline & 13 & 288.540 & 20,6 \\
\hline & 14 & 152.168 & 10,9 \\
\hline & 15 & 290.208 & 20,7 \\
\hline \multirow{3}{*}{ Desplazarse } & 16 & 428.802 & 30,7 \\
\hline & 17 & 622.091 & 44,5 \\
\hline & 18 & 537.857 & 38,5 \\
\hline \multirow{3}{*}{ Utilizar brazos y manos } & 19 & 517.127 & 37,0 \\
\hline & 20 & 425.449 & 30,4 \\
\hline & 21 & 366.204 & 26,2 \\
\hline \multirow{3}{*}{ Desplazarse fuera del hogar } & 22 & 1.008 .111 & 72,1 \\
\hline & 23 & 1.103 .026 & 78,9 \\
\hline & 24 & 497.098 & 35,5 \\
\hline \multirow{4}{*}{ Autocuidado } & 25 & 600.311 & 42,9 \\
\hline & 26 & 282.607 & 20,2 \\
\hline & 27 & 509.414 & 36,4 \\
\hline & 28 & 180.182 & 12,9 \\
\hline \multirow{5}{*}{ Realizar tareas del hogar } & 29 & 927.547 & 66,3 \\
\hline & 30 & 715.279 & 51,1 \\
\hline & 31 & 861.675 & 61,6 \\
\hline & 32 & 1.026 .025 & 73,4 \\
\hline & 33 & 686.156 & 49,1 \\
\hline \multirow{3}{*}{ Relaciones con otras personas } & 34 & 96.318 & 6,9 \\
\hline & 35 & 279.077 & 20,0 \\
\hline & 36 & 359.025 & 25,7 \\
\hline Total de la población* & & 1.398 .767 & \\
\hline
\end{tabular}

"No se corresponde con la suma de la columna número de personas. Una misma persona puede sufrir más de una discapacidad.

Fuente: elaboración propia a partir de EDDES.

dientes 468.000 tendrían algún tipo de ayuda en el sistema español, la situación contraria únicamente se da en 8.000 personas. Otro resultado importante es que todos los que alcanzan el GIR 1 están dentro del Grado III español; sin embargo, 48.501 personas situadas en el máximo español perde- rían sus ayudas si se aplicase el esquema francés.

Tras la simple comparación entre los resultados de la clasificación del sistema español y los otros dos sistemas considerados, se estudia la posible existencia de simi- 
Tabla 5

Comparación entre el baremo español y el baremo alemán

\begin{tabular}{|l|c|c|c|c|c|c|}
\hline \multirow{2}{*}{\multicolumn{2}{|l|}{ Baremo español }} & \multicolumn{5}{|c|}{ Baremo alemán } \\
\cline { 3 - 7 } \multicolumn{2}{|l|}{0} & $\mathbf{0}$ & N I & N II & N III & Total general \\
\hline \multirow{2}{*}{ G I } & N1 & 490.302 & 122.493 & 8.644 & 854 & 622.292 \\
\cline { 2 - 7 } & N2 & 20.878 & 143.277 & 17.884 & 0 & 273.766 \\
\hline \multirow{2}{*}{ G II } & N1 & 15.698 & 61.027 & 28.406 & 0 & 110.311 \\
\cline { 2 - 7 } & N2 & 6.568 & 47.686 & 66.822 & 213 & 61.575 \\
\hline \multirow{2}{*}{ G III } & N1 & 5.647 & 15.675 & 39.119 & 4.510 & 91.841 \\
\cline { 2 - 7 } & N2 & 1.550 & 15.235 & 66.449 & 73.049 & 108.776 \\
\hline \multicolumn{2}{|l}{ Total general } & 653.247 & 416.697 & 22.873 & 78.626 & 1.398 .767 \\
\hline
\end{tabular}

NOTA: el "0" en el baremo español significa que no llega a 25 puntos y en el baremo alemán recoge a los dependientes sin derecho a recibir ayudas de acuerdo con la escala alemana.

Fuente: elaboración propia.

Tabla 6

Comparación entre el baremo español y el baremo francés

\begin{tabular}{|c|c|c|c|c|c|c|c|c|c|}
\hline \multirow{2}{*}{\multicolumn{2}{|c|}{ Baremo español }} & \multicolumn{7}{|c|}{ GIR } & \multirow{2}{*}{$\begin{array}{c}\text { Total } \\
\text { general }\end{array}$} \\
\hline & & 1 & 2 & 3 & 4 & 5 & 6 & $\mathbf{0}$ & \\
\hline \multicolumn{2}{|l|}{0} & 0 & 1.325 & 461 & 6.145 & 43.229 & 403.391 & 167.741 & 622.292 \\
\hline \multirow[t]{2}{*}{ G I } & N1 & 0 & 418 & 1.694 & 19.018 & 63.752 & 124.695 & 64.189 & 273.766 \\
\hline & $\mathrm{N} 2$ & 0 & 1.545 & 2.536 & 31.913 & 20.968 & 29.914 & 23.436 & 110.311 \\
\hline \multirow[t]{2}{*}{ G II } & N1 & 0 & 4.306 & 8.511 & 51.431 & 10.119 & 23.477 & 32.361 & 130.206 \\
\hline & $\mathrm{N} 2$ & 0 & 5.879 & 3.866 & 24.350 & 2.756 & 8.655 & 16.069 & 61.575 \\
\hline \multirow[t]{2}{*}{ G III } & N1 & 592 & 19.657 & 9.646 & 36.701 & 1.813 & 6.130 & 17.303 & 91.841 \\
\hline & $\mathrm{N} 2$ & 12.113 & 39.968 & 12.265 & 21.175 & 2.029 & 607 & 20.619 & 108.776 \\
\hline \multicolumn{2}{|c|}{ Total general } & 12.705 & 73.098 & 38.978 & 190.733 & 144.666 & 596.869 & 341.719 & 1.398 .767 \\
\hline
\end{tabular}

NOTA: el "0" en el baremo español significa que no llega a 25 puntos y en el GIR que no llega a alcanzar el nivel 6.

Fuente: elaboración propia.

litudes entre niveles de cada uno de los esquemas objeto de análisis. Para ello se utiliza el análisis simple de correspondencias. Por lo que se refiere a la comparación entre el modelo español y el alemán se obtiene que el coeficiente de correlación de Spearman entre las categorías es de 0,724 y el coeficiente de Kendall es de 0,658. Ambos estadísticos sugieren una elevada correspondencia entre ambos esquemas. De hecho, al calcular el estadístico ji-cuadrado del contraste de independencia entre los pares de ambos sistemas se rechaza claramente la hipótesis de independencia, pues se obtiene una $p<0,0001$. La figura 1 recoge los dos primeros factores del análisis de correspondencias, los cuales representan el $91,72 \%$ de la información $(57,29 \%$ el pri- mero y $34,44 \%$ el segundo). Es evidente que el primer factor está directamente correlacionado con la intensidad de la ayuda a la que se accedería, cuanto mayor es el valor del factor mayor es la ayuda recibida. El segundo factor parece discriminar las situaciones extremas (máxima ayuda o ninguna) de las intermedias. Tras el análisis de las inercias asociadas a cada categoría se obtiene que en el sistema alemán la categoría peor representada por los dos factores es N1 $(25,50 \%)$, situándose el resto por encima del $90 \%$. En el caso español los peores representados por los factores son los dos niveles del Grado I (27,60\% y 54,39\%, respectivamente). Del estudio de la asociación entre categorías de dependientes y factores resultan significativas las referidas a los 


\section{Figura 1}

Resultados del análisis de correspondencia que compara los sistemas de dependencia español y alemán

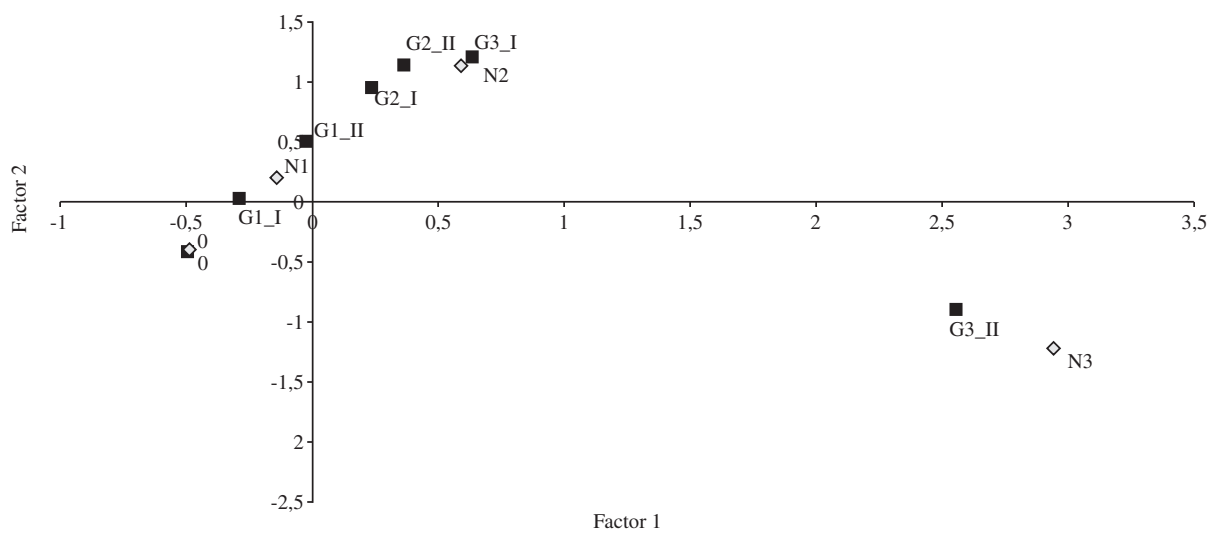

$\diamond$ Alemania

- España

Figura 2

Resultados del análisis de correspondencia que compara los sistemas de dependencia español y francés

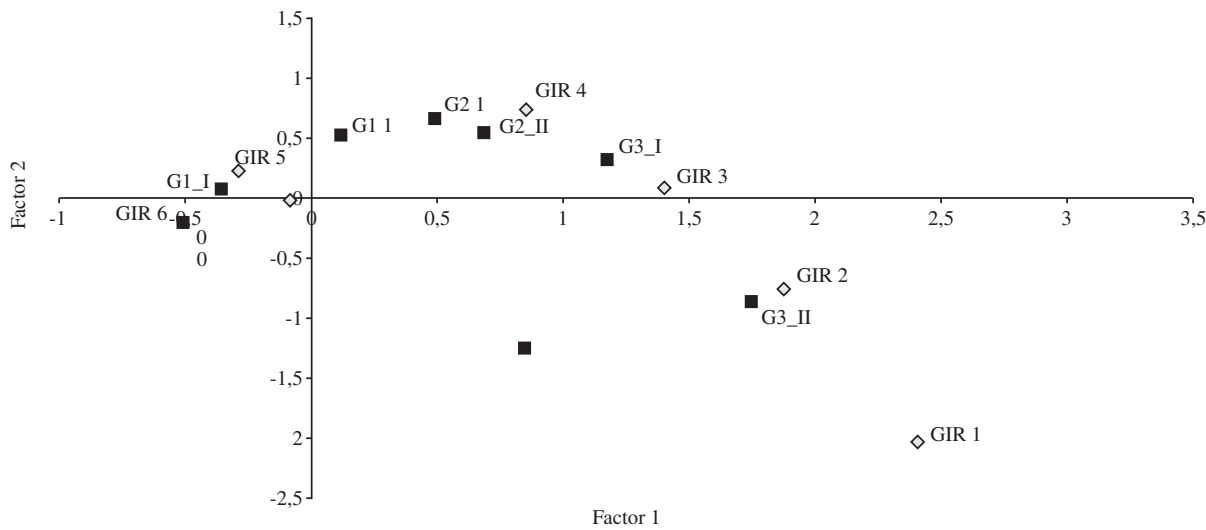

$\diamond$ Francia

- España

grupos denominados como " 0 " -dependientes sin ayuda- y los niveles N2 y N3 alemanes con los grados II y III españoles.

Por lo que respecta a la comparación entre el sistema español y el francés, tras reordenar las categorías de este último de menor -0- a mayor -GIR 1- se obtiene que el coeficiente de Spearman es de 0,444 y el coeficiente de Kendall es 0,389, los cuáles están muy por debajo de los obtenidos en la relación con el sistema alemán. El estadístico ji-cuadrado tiene asociado una $\mathrm{p}<0,0001$, lo cual indica que existe 
correlación significativa entre ambos sistemas. La figura 2 recoge los dos primeros factores del análisis de correspondencias, los cuales representan el $92,07 \%$ de la información $(70,30 \%$ el primero y $21,78 \%$ el segundo). La interpretación de los factores coincide con la descrita anteriormente para el caso alemán. El análisis de las inercias asociadas a cada categoría indica que en el sistema francés los niveles peor representados son el "0" y el GIR $5(63,38 \%$ y $26,28 \%$ respectivamente), situándose el resto por encima del $90 \%$. En este caso, sólo ambos niveles del Grado I español tienen un porcentaje de representación inferior al $80 \%$. A partir de las contribuciones parciales de los factores a la inercia en cada categoría se obtiene que en el sistema español las personas dependientes de Grado III nivel 2 tienden a coincidir con GIR 2 y los de Grado III nivel 1 con GIR 3. Por otro lado, las personas dependientes de Grado II se asocian a GIR 4 y, por último, las personas dependientes sin derecho a ayuda del sistema español se asocian a GIR 6. Para el Grado I español, y para GIR 5 y "0" del francés no se detectan asociaciones significativas. Por último, la categoría GIR 1 del sistema francés puede asociarse al Grado III nivel 2 español, aunque debido a la poca masa de GIR 1, en el gráfico aparece a una distancia mayor.

\section{DISCUSIÓN}

Los resultados de este trabajo muestran que el país que incorpora mayor número de discapacidades del CIDDM-1980 en su baremo es España, con un total de 17, le sigue Francia con 14 y, por último, se sitúa Alemania con 12. Al margen de las diferencias en su número, existen similitudes respecto a las discapacidades consideradas en los tres sistemas analizados, puesto que los tres países incluyen discapacidades relacionadas con "desplazarse dentro y fuera del hogar" así como los "autocuidados". Ade- más, por un lado, España y Alemania incorporan discapacidades ligadas a la posibilidad de "realizar tareas del hogar" y, por otro, España y Francia incluyen discapacidades relacionadas con el "aprendizaje". Estos resultados podrían indicar que el baremo español se ha diseñado como una mezcla algo ampliada de los dos anteriores. Finalmente, destacar que el aspecto que diferencia el baremo francés del resto es el hecho de incorporar las discapacidades relacionadas con "comunicarse".

En este trabajo se ha analizado el impacto que pueden tener los diferentes baremos de valoración de la dependencia sobre un mismo conjunto de población (la española) aplicando tres sistemas: el alemán, el francés y el español -el suyo propio-. Para aplicar los tres sistemas ha habido que adaptar las discapacidades que contempla la EDDES a cada uno de los baremos analizados.

Se observa que tanto el número como la distribución de personas dependientes según tramos son diferentes en función del criterio o sistema empleado en cada país. Los resultados sugieren que como la relación de discapacidades requeridas y ciertas exigencias en materia de tiempos de cuidado o edad en cada uno de ellos es distinta, el número de potenciales beneficiarios también lo es. Se obtiene que la escala más generosa es la española, mientras que en el otro extremo se sitúa la francesa, si bien es cierto que ésta limita las ayudas a personas de 60 años o más. Además, tal y como se comentó anteriormente, en Francia se consideran las discapacidades relacionadas con la comunicación que afectan a un porcentaje muy bajo de la población española. Sin embargo, no incluyen las discapacidades asociadas a la realización de tareas del hogar (incluidas por Alemania y España) que afectan a más de la mitad de la población dependiente. Estas circunstancias pueden ayudar a explicar las notables diferencias entre los resultados obtenidos. 
Las consecuencias de la aplicación de uno u otro baremo son muy diferentes: con el sistema español más de 776.000 personas recibirían ayudas, mientras que con el francés, esta cifra se recorta a algo más de 315.000. Por su parte, los resultados con la escala alemana son más próximos a los de la escala española. Aun siendo importante el resultado obtenido, por su incidencia sobre la financiación de la atención a estas personas, no hay que olvidar que las discapacidades asociadas a los datos utilizados no tienen una traducción directa a los baremos aquí aplicados, ni siquiera al español. Es importante destacar que la selección de discapacidades para simular los resultados de cada uno de los sistemas de valoración se ha realizado de forma muy cuidadosa, intentando reproducir al máximo las definiciones originales. De todos modos, podría haber cierta subjetividad en la selección de las discapacidades ligadas a cada uno de los baremos analizados. Ante ello, consideramos que la elección de unas u otras discapacidades podrían modificar sensiblemente los resultados obtenidos, aunque las principales conclusiones tenderían a ser las mismas.

Considerando los aspectos económicos de la mayor incidencia de la dependencia en un contexto de envejecimiento de la población ${ }^{34}$, diversos trabajos se centran en comparar los sistemas de financiación de la dependencia utilizados en distintos paí$\operatorname{ses}^{35,36}$. Sin embargo, el análisis del sistema de financiación de la dependencia en un determinado país no puede estar separado del concepto y valoración de la dependencia en dicho país. Así, partiendo de los resultados obtenidos, y como continuidad a este trabajo en la línea de algunos autores ${ }^{26}$, resulta de utilidad la estimación de los costes asociados a los cuidados requeridos por las personas en situación de dependencia a cualquier edad y su comparación con las dotaciones de recursos necesarias según se aplique el criterio francés, alemán o el español. Obviamente, como el mayor número de personas se registra al aplicar los criterios de la ley española, con éste sistema los costes serán mayores. Además, cabe profundizar en el estudio específico de aquellas personas que según un sistema tienen derecho a ayudas y dejan de tenerlas según otro criterio, para determinar qué factores influyen en que se produzcan estas situaciones. Enlazando con esto último, es necesario recordar que existen trabajos centrados en analizar los perfiles socioeconómicos de los dependientes ${ }^{19,37}$. Siguiendo esta línea de trabajo, tendría sentido analizar si existen diferencias significativas entre los perfiles socioeconómicos de las personas dependientes, con derecho a ayuda por parte de la administración, en función del baremo nacional aplicado.

\section{BIBLIOGRAFÍA}

1. Comisión Europea. Communication from the Commission, Confronting demographic change: a new solidarity between the generations. COM (2005) 95 final. Bruselas: Comisión Europea; 2005.

2. WHO. Highlights on health in Germany. Geneva, World Health Organization 2004a. Citado en diciembre de 2007. Disponible en: http://www.euro.who.int/document/e88527.pdf.

3. WHO. Highlights on health in France. Geneva, World Health Organization 2004b. Citado en octubre de 2008. Disponible en: http://www.euro.who.int/document/e88547.pdf.

4. WHO. Highlights on health in Spain. Geneva, World Health Organization 2004c. Citado en diciembre de 2007. Disponible en: http://www.euro.who.int/document/chh/spa_highlights.pdf.

5. Department of Economic and Social Affairs, Population Division. World Population Ageing: 1950-2050. New York: United Nations; 2001. Citado en octubre de 2008. Disponible en: http:// www.un.org/esa/population/publications/worldageing 19502050/

6. Iacob IN y Rodríguez S. A European perspective of services organisation for dependent elderly people care. Rennes (France): ENSP; 2007. 
7. Wiener JM, Tilly J y Cuellar AE. ConsumerDirected Home Care in the Netherlands, England and Germany, Washington (DC): Public Policy Institute; 2003.

8. Zweifel P, Felder S y Werblow, A. Population ageing and health care expenditure: New evidence for the "red herring". The Geneva Papers on Risk and Insurance Issues and Practice 2004; 29(4): 652-666.

9. Courbage C y Costa-Font J. On Health, Ageing and Insurance. The Geneva Papers on Risk and Insurance Issues and Practice 2006; 31: 551-556.

10. Economic Policy Committee. Budgetary challenges posed by ageing populations: the impact on public spending on pensions, health and long-term care for the elderly and possible indicators of the long term sustainability of public finances. European Economy, 2001 Reports and Studies 04-01.

11. Economic Policy Committee. The 2005 EPC projections of age-related expenditure: Agreed underlying assumptions and projection methodologies. European Economy, 2005 Special Reports $4 / 2005$.

12. Kotlikoff LJ y Hagist C. Who's going broke? Comparing healthcare costs in ten OECD countries; 2005 NBER Working Paper 11833.

13. Abio Roig G. Population Ageing and the Sustainability of the Spanish National Health System: Some Financial Policy Alternatives. The Geneva Papers on Risk and Insurance Issues and Practice 2006; 31: 557-580.

14. Otero A, Zunzunegui MV, Rodríguez-Laso A, Aguilar MD y Lázaro P. Volumen y tendencias de la dependencia asociada al envejecimiento en la población española, Rev. Esp. Salud Pública 2004; 78: 201-213.

15. Consejo de Europa. Recomendación n ${ }^{\circ}(98) 9$ del Comité de Ministros a los Estados miembros relativa a la dependencia. CE num (98)9, 1998.

16. European Observatory. Health Care Systems in Transition. Germany: European Observatory; 2000.

17. Journal Officiel. Loi 2001-647 relative à la prise en charge de la perte d'autonomie des personnes âgées et à l'allocation personnalisée d'autonomie. J.O. num. 167, 21/7/2001.

18. Boletín Oficial del Estado. Ley 39/ 2006 de Promoción de la Autonomía Personal y Atención a las personas en situación de dependencia. BOE num. $299,15 / 12 / 2006$.
19. Albarrán I y Alonso P. Clasificación de las personas dependientes a partir de la Encuesta de Discapacidades, Deficiencias y Estado de Salud de 1999. Rev. Esp. Salud Pública 2006; 80: 349-360.

20. Lea RD, Etheredge GD, Freeman JN y Lloyd WB. Familial Disability Patterns in Individuals with Chronic Work-related Spine Injury/illness. Spine 2003; 28(19): 2292-2297.

21. Zunzunegui MV y Béland F. La salud de las personas mayores de Leganés, Rev. Gerontol. 1995; 5: 245-258.

22. Lesemann F y Martin C. (dir.). Les personnes âgées dépendantes. Dépendance, soins et solidarités familiales. Comparaisons internationales. Paris: La Documentation Française; 1993.

23. OECD. Protéger les personnes âgées dépendantes: des politiques en mutation. Etudes de Politique Sociale 1996; 19.

24. Jacobzone S. Les perspectives de prise en charge de la dépendance au niveau international. Retraite et Société 1999; 25 : 37-65.

25. Assous L, Ralle P. La prise en charge de la dépendance des personnes âgées: une comparaison internationale. Etudes et Résultats 2000; 74 (July). Citado en diciembre de 2007. Disponible en: http://www.sante.gouv.fr/drees/etude-resultat/erpdf/er074.pdf.

26. Guillén M y Blay D. Coste de atención a la dependencia en España y comparación con los sistemas francés y alemán. Revista Española de Seguros 2006; 125: 145-160.

27. Le Bihan B y Martin CA. A comparative study of care systems towards frail elderly people: Germany, Spain, France, Italy, United Kingdom and Sweden. Social Policy and Administration 2006; 40(1): 26-46.

28. Cabrero J. Indicadores de discapacidad en la Encuesta de Discapacidades, Deficiencias y Estado de Salud. Rev. Esp. Salud Pública 2007; 81: 167-181.

29. Alegre A, Ayuso M, Guillén M, Monteverde M y Pociello E. Tasa de dependencia de la población española no institucionalizada y criterios de valoración. Rev. Esp. Salud Pública 2005; 79: 351-363.

30. Ministerio de Trabajo y Asuntos Sociales. Libro Blanco de la Dependencia; 2005. Citado en diciembre de 2007. Disponible en: http://www.tt. mtas.es/periodico/serviciossociales/200501/libro_ blanco_dependencia.htm 
31. Boletín Oficial del Estado. Real Decreto 504/2007, de 20 de abril, por el que se aprueba el baremo de valoración de la situación de dependencia establecido por la Ley 39/2006, de 14 de diciembre, de promoción de la autonomía personal y atención a las personas en situación de dependencia. BOE num. 96, 21/04/2007.

32. Benzécri, J.-P. Correspondence Analysis Handbook. New York: Marcel Dekker; 1996.

33. Greenacre M.J. Theory and Applications of Correspondence Analysis. London: Academic Press; 1984.

34. Puga D. La dependencia de las personas con discapacidad: entre lo sanitario y lo social, entre lo privado y lo público. Rev Esp Salud Pública 2005; 79: 327-330.

35. Kalsson M, Mayhew L, Plumb R y Rickayzen B. An International Comparison of Long-Term Care
Arrangements: An Investigation into the Equity, Efficiency and sustainability of the Long-Term Care Systems in Germany, Japan, Sweden, the United Kingdom and the United States. London: Faculty of Actuarial Science and Statistics; 2004 Actuarial Research Paper No. 156.

36. Comal-Herrera A y Wittenberg, R. editores. European Study of Long-Term Care Expenditure: Investigating the Sensitivity of Future Long-Term Care Expenditure in Germany, Spain, Italy and United Kingdom to Changes in Assumptions about Demography, Dependency, Informal Care, Formal Care and Unit Costs. Report to the European Commission: Employment and Social Affairs DG; 2003 PSSRU discussion paper No. 1840.

37. Bermúdez L, Bolancé C, Mustafa-Gondolbeu K y Guillén, M. Tipologías sociodemográficas de individuos con dependencia en España y su supervivencia en estado de salud. Rev Esp Geriatr Gerontol. 2008 ; 43: 19-31. 